Research Paper

\title{
Urinary Orosomucoid A Potential Marker Of Inflammation In Psoriasis
}

\author{
Péter Kustán¹,2, Tamás Kőszegi ${ }^{1,2}$, Attila Miseta1,2, Iván Péter³, Zénó Ajtay³, István Kiss4, Balázs Németh³,4,凹 \\ 1. Department of Laboratory Medicine, University of Pécs Medical School, Pécs, Hungary \\ 2. János Szentágothai Research Centre, University of Pécs, Pécs, Hungary \\ 3. Zsigmondy Vilmos SPA Hospital, Harkány, Hungary \\ 4. Department of Public Health Medicine, University of Pécs Medical School, Pécs, Hungary \\ $\square$ Corresponding author: Balázs Németh, MD, Department of Public Health Medicine, University of Pécs Medical School, Pécs, Hungary, Szigeti u. 12 . H-7624 \\ Pécs, Hungary. Tel: +36 72 536394/36037, E-mail: balazs.nemeth@aok.pte.hu
}

(1) Ivyspring International Publisher. This is an open access article distributed under the terms of the Creative Commons Attribution (CC BY-NC) license (https:// creativecommons.org/licenses/by-nc/4.0/). See http://ivyspring.com/terms for full terms and conditions.

Received: 2018.02.23; Accepted: 2018.05.31; Published: 2018.07.13

\begin{abstract}
Background: Psoriasis is one of the most common chronic, life-long dermatologic diseases, which has considerable negative effects on quality of life. Psoriasis is considered as a systemic inflammatory disease, thus acute phase proteins such as C-reactive protein (CRP) and orosomucoid (ORM) have been shown to play a role in its pathophysiology. This study was aimed to compare CRP, serum ORM (se-ORM) and urinary ORM (u-ORM) levels of psoriatic patients to healthy individuals.

Methods: 87 psoriatic patients and 41 healthy individuals were enrolled. Simultaneously obtained venous blood and spot urine samples were analysed. High sensitivity CRP and se-ORM levels were determined by routine procedures on automated analyzers. Urinary ORM was measured by a novel automated turbidimetric assay. U-ORM was referred to urinary creatinine (u-ORM/u-CREAT, $\mathrm{mg} / \mathrm{mmol}$ ).

Results: Significantly higher hsCRP $(p<0.001)$ and $u-O R M / u-C R E A T(p=0.001)$ levels were found among psoriatic patients compared to controls. No significant differences were found between the groups regarding se-ORM levels. HsCRP, se-ORM and u-ORM/u-CREAT levels were significantly higher in patients with severe psoriasis than in mild and moderate cases $(p<0.05)$.

Conclusion: As a highly sensitive, easily available biomarker u-ORM shows itself capable of becoming a new inflammatory marker in psoriasis providing clinically useful information on disease severity.
\end{abstract}

Key words: Psoriasis; Orosomucoid; Urine, Biomarker, Inflammation

\section{Introduction}

Psoriasis is one of the most common chronic, lifelong dermatologic diseases [1]. Due to physical and psychical symptoms, psoriasis has a considerable negative effect on quality of life [2]. Furthermore, it has been shown that psoriasis is often associated with higher risk of developing obesity, insulin resistance, diabetes, depression, inflammatory bowel disease and some malignancies [3, 4]. Moreover, psoriasis is often associated with adverse cardiovascular outcomes [5]. Several factors have been identified to play a role in the development of psoriasis. Genetical background and the onset of general risk factors (stress, obesity, smoking and alcohol consumption) can lead to an excessive activation of the immune system, which has been identified as a key factor in the development of psoriasis [6-8]. Psoriasis is considered as a systemic disease, thus numerous markers of systemic inflammation have been investigated in psoriatic patients.

One of the most frequently used and widely available markers of inflammation is the $\mathrm{C}$ - reactive protein (CRP). Several studies found increased CRP 
levels in psoriatic patients; some of these studies even suggested that CRP could be used as a marker of psoriasis severity [9- 12]. These findings were confirmed by a meta-analysis published by Dowlatshahi et al. However, the meta-regression did not show significant association between CRP levels and psoriasis severity [13].

Orosomucoid (ORM) or a-1-acid glycoprotein is another major acute phase protein mainly produced by the liver, accounting for approximately $0.5-1.2 \mathrm{~g} / \mathrm{L}$ of serum proteins [14]. Serum ORM (se-ORM) levels may increase a few fold in several acute and chronic disorders in response to systemic inflammation. Elevated se-ORM has been found in patients suffering from infections, malignancies and autoimmune diseases such as rheumatoid arthritis, inflammatory bowel disease and psoriasis $[15,16]$. It appears that se-ORM is a non-specific and non-sensitive marker of inflammation, thus its clinical value can be questioned. Although recent studies have demonstrated urinary orosomucoid (u-ORM) as a more sensitive, non- invasive biomarker of inflammatory activation than se-ORM, the clinical utility of u-ORM is yet to be explored [17]. Normally urinary excretion of ORM is low and u-ORM concentrations account for only a few $\mathrm{mg} / \mathrm{L}$ (0.01-0.3 $\mathrm{mg} / \mathrm{mmol}$ if referred to urinary creatinine), however, increased u-ORM levels have been described in certain acute and chronic diseases [18-21]. The elevation of u-ORM seems to be associated with systemic inflammation and endothelial dysfunction, which are also considerable factors in the patomechanism of psoriasis $[2,4,6,7,17,22]$.

To the best of our knowledge, no study has been performed to investigate $\mathrm{u}$-ORM levels in psoriatic patients. This study was aimed to investigate conventional inflammatory markers as CRP and se-ORM and a novel promising biomarker u-ORM in psoriatic patients and to examine their clinical utility.

\section{Materials and Methods}

\section{Study participants}

The present study was performed at our ISO 9001 accredited Dermatology Rehabilitation Unit between November 2016 and July 2017. Patients suffering from psoriasis with skin lesions were investigated. Exclusion criteria were impaired renal function $\left(\right.$ eGFR $\left.<60 \mathrm{ml} / \mathrm{min} / 1.73 \mathrm{~m}^{2}\right)$, acute inflammations, autoimmune disorders (rheumatoid arthritis, systemic lupus erythematosus, inflammatory bowel disease), any kind of biological antipsoriatic treatment and withdrawal of consent. Healthy volunteers without any kind of acute or chronic diseases were enrolled as controls.
The study protocol was approved by the Regional Ethics Committee of University of Pécs, Hungary (Permission No.: 5919/2.), in accordance with the 2008 Helsinki declaration. Written informed consent was obtained from every patient and control.

Psoriasis severity was measured by the Psoriasis Area and Severity Index (PASI) and patients were categorized based on PASI as mild (PASI $<7$ ) moderate (PASI 7-12) and severe (PASI $>12$ ) groups [23]. To rule out interobserver errors PASI was assessed by the same dermatologist in all cases.

\section{Biochemical measurements}

Venous blood and midstream first morning urine samples were simultaneously obtained from every patient and control. Laboratory tests were performed at the university's clinical laboratory (accreditation no.: NAH-1-1553/2016). Conventional inflammatory parameters (high sensitivity C-reactive protein (hsCRP), white blood cell count (WBC), se-ORM) were measured by routine procedures. U-ORM was determined by an automated turbidimetric assay using ORM immunoparticles (Dako A/S, Glostrup, Denmark) [18]. Since spot urine specimens were analyzed, u-ORM levels were divided by urinary creatinine (u-ORM/u- CREAT, $\mathrm{mg} / \mathrm{mmol}$ ) to avoid the influence of the variations of urinary solute concentrations caused by different hydration states.

\section{Statistical analysis}

Statistical analysis was performed with IBM SPSS Statistics for Windows, Version 22 (IBM Corp., New York, NY, USA). Values were compared by Mann-Whitney U test and Kruskal- Wallis test. To reveal correlations, the Spearman's test was used. Data are presented as median and interquartiles. All p-values less than 0.05 were considered statistically significant.

\section{Results}

87 psoriatic patients and 41 healthy individuals were enrolled in the present study. The clinical characteristics of the involved patients and controls are presented in Table 1.

Table 1. Clinical characteristics of the involved individuals

\begin{tabular}{llll}
\hline & Controls (n=41) & Patients (n=87) & p-value \\
\hline Age, years & $44(30-53)$ & $66(61-70)$ & $<0.001$ \\
Male, n (\%) & $17(41.5)$ & $47(54.0)$ & - \\
BMI, kg/m2 & $28.2(26.1-31.9)$ & $29.7(26.4-33.7)$ & n.s \\
Diabetes type 2, n (\%) & - & $23(26.4)$ & - \\
Hypertension, n (\%) & - & $69(79.3)$ & - \\
COPD, n (\%) & - & $5(5.7)$ & - \\
Malignancies, n (\%) & - & $0(0)$ & - \\
\hline \\
BMI: Body mass index; COPD: chronic obstructive pulmonary disease; n.s: not \\
significant.
\end{tabular}




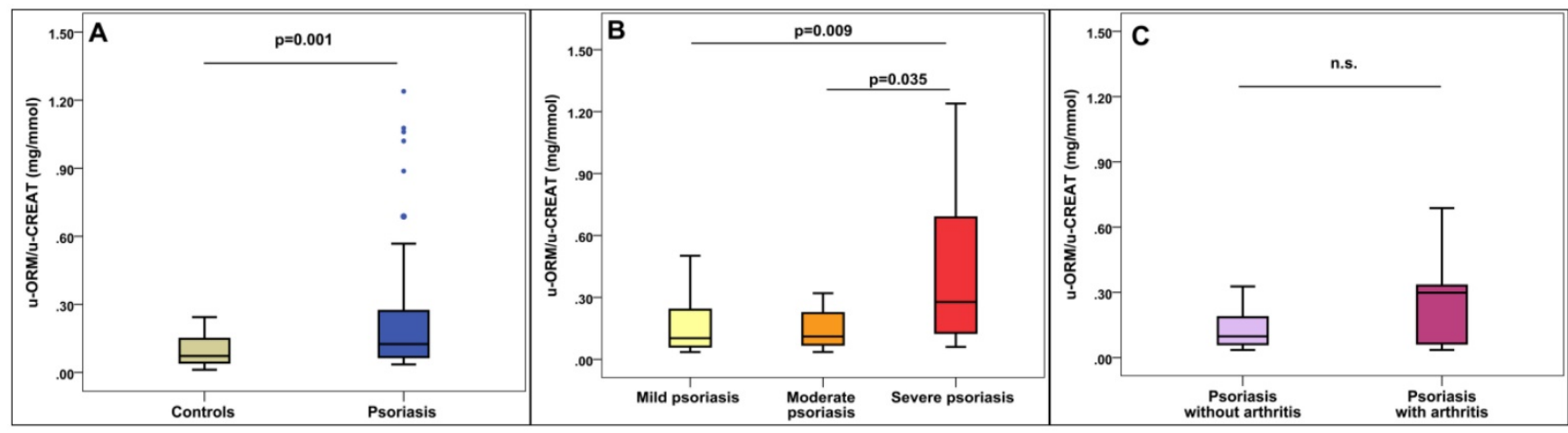

Figure 1. U-ORM/u-CREAT levels in psoriatic patients. A: u-ORM/u-CREAT levels in psoriasis compared to healthy controls. B: u-ORM/u-CREAT levels based on Psoriasis Area and Severity Index (PASI), mild: PASI<7, moderate: PASI:7-12, severe: PASI>12. C: u-ORM/u-CREAT levels in psoriatic arthritis, n.s.: not significant ( $P=0.23$ )

The mean PASI of the patients was $6.52 \pm 7.11$. Based on PASI, $64.4 \%$ of the patients had mild psoriasis, $20.7 \%$ had moderate and $14.9 \%$ had severe psoriasis. $33.3 \%$ of the patients had positive family history of psoriasis. The mean onset of psoriasis was $26.7 \pm 17.1$ years. 49 patients $(56.3 \%)$ had psoriasis for more than 30 years. Psoriatic arthritis occurred in $8 \%$ of the patients $(n=7)$.

Significantly higher hsCRP levels were found among psoriatic patients when comparing the hsCRP levels of the patients to that of the controls $(p<0.001)$. Significantly higher $\mathrm{u}-\mathrm{ORM} / \mathrm{u}-\mathrm{CREAT}$ levels were found in patients compared to controls $(p=0.001$, Figure 1A). On the other hand, se-ORM and WBC did not show significant differences. Detailed results of the biomarker measurements are shown in Table 2.

Table 2. Laboratory parameters of the involved individuals

\begin{tabular}{llll}
\hline & Controls $(\mathbf{n}=41)$ & Patients $(\mathbf{n}=87)$ & p-value \\
\hline se-CREAT, $(\mu \mathrm{mol} / \mathrm{L})$ & $74(62-81)$ & $77(68-84)$ & $\mathrm{n} . \mathrm{s}$ \\
WBC, $(\mathrm{G} / \mathrm{L})$ & $6.4(4.9-7.6)$ & $5.9(5.1-7.3)$ & $\mathrm{n} . \mathrm{s}$ \\
$\mathrm{hsCRP},(\mathrm{mg} / \mathrm{L})$ & $0.90(0.50-1.80)$ & $3.46(1.12-6.29)$ & $<0.001$ \\
se-ORM, (g/L) & $0.80(0.70-0.90)$ & $0.79(0.68-0.98)$ & $\mathrm{n} . \mathrm{s}$ \\
u-TP, $(\mathrm{mg} / \mathrm{L})$ & $60(40-80)$ & $60(40-80)$ & $\mathrm{n} . \mathrm{s}$ \\
u-CREAT, $(\mathrm{mmol} / \mathrm{L})$ & $11.50(5.92-15.34)$ & $8.64(6.19-12.50)$ & $\mathrm{n} . \mathrm{s}$ \\
u-ORM, (mg/L) & $0.82(0.33-1.14)$ & $1.08(0.57-2.38)$ & 0.004 \\
u-ORM/u-CREAT, & $0.07(0.04-0.15)$ & $0.12(0.07-0.28)$ & 0.001 \\
(mg/mmol) & & &
\end{tabular}

Median (interquartiles) data are presented. CREAT: creatinine, WBC: white blood cell count; hsCRP: high sensitivity C-reactive protein; se-ORM: serum orosomusoid; u-TP: urinary total protein; u-ORM: urinary orosomucoid u-ORM/u-CREAT: urinary orosomucoid urinary creatinine ratio; n.s: not significant.

Regarding psoriasis severity, significantly higher hsCRP, se-ORM and u-ORM/u-CREAT levels were found in patients with severe psoriasis compared to patients with mild and moderate symptoms. However, none of the investigated parameters showed significant differences between mild and moderate psoriasis (Table 3 and Figure 1B).

Regarding complications, higher $\mathrm{u}-\mathrm{ORM} / \mathrm{u}-$ CREAT ratio was found in patients with psoriatic arthritis compared to patients without joint involvement $(0.30 \quad(0.04-0.35) \quad \mathrm{mg} / \mathrm{mmol} \quad$ vs $\quad 0.10$
(0.06-0.20) $\mathrm{mg} / \mathrm{mmol})$, however, the differences did not meet the statistical significance $(p=0.23$, Figure 1C). Neither hsCRP nor se-ORM was elevated in psoriatic arthritis patients (Table 4).

Table 3. Parameters of psoriatic patients based on the Psoriasis Area and Severity Index

\begin{tabular}{|c|c|c|c|c|}
\hline & $\begin{array}{l}\text { Mild: PASI }<7 \\
(n=56)\end{array}$ & $\begin{array}{l}\text { Moderate: } \\
\text { PASI:7-12 } \\
(\mathrm{n}=18)\end{array}$ & $\begin{array}{l}\text { Severe: } \\
\text { PASI }>12(n=13)\end{array}$ & p-value \\
\hline WBC, (G/L) & $6.1(5.1-7.3)$ & $5.2(4.6-7.7)$ & $6.7(5.2-7.8)$ & n.s. \\
\hline hsCRP, (mg/L) & $3.03(1.05-4.70)$ & $2.49(0.86-3.70)$ & 7.78 (5.19-11.79) & $0.002^{a, b}$ \\
\hline se-ORM, (g/L) & $0.78(0.69-0.98)$ & $0.75(0.61-0.92)$ & $0.98(0.84-1.12)$ & $0.028 b$ \\
\hline $\mathrm{u}-\mathrm{TP},(\mathrm{mg} / \mathrm{L})$ & $56(40-75)$ & $50(40-70)$ & $60(40-90)$ & n.s. \\
\hline $\begin{array}{l}\text { u-CREAT, } \\
(\mathrm{mmol} / \mathrm{L})\end{array}$ & $8.54(6.68-12.22)$ & 9.77 (7.18-12.23) & $8.03(4.71-12.41)$ & n.s. \\
\hline $\mathrm{u}$-ORM, (mg/L) & $1.08(0.60-1.65)$ & $0.82(0.54-2.64)$ & $2.72(1.06-4.92)$ & $0.048^{a}$ \\
\hline $\begin{array}{l}\text { u-ORM/u-CREAT, } \\
(\mathrm{mg} / \mathrm{mmol})\end{array}$ & $0.10(0.06-0.24)$ & $0.11(0.07-0.22)$ & $0.28(0.13-0.69)$ & $0.008^{a, b}$ \\
\hline \multicolumn{5}{|c|}{$\begin{array}{l}\text { Median (interquartiles) data are presented. WBC: white blood cell count; hsCRP: } \\
\text { high sensitivity C-reactive protein; se-ORM: serum orosomucoid; u-TP: urinary } \\
\text { total protein; u-CREAT: urinary creatinine; u-ORM: urinary orosomucoid; n.s: not } \\
\text { significant. Superscript lowercase letters show post-hoc analysis: a: mild vs } \\
\text { severe: } p<0.05 \text {, b: moderate vs severe: } p<0.05 \text {. Kruskal-Wallis test with post hoc } \\
\text { analyses was used for comparison. }\end{array}$} \\
\hline
\end{tabular}

Table 4. Parameters in psoriatic arthritis

\begin{tabular}{llll}
\hline & $\begin{array}{l}\text { Patients without } \\
\text { arthritis }(\mathbf{n}=\mathbf{8 0})\end{array}$ & $\begin{array}{l}\text { Patients with } \\
\text { arthritis (n=7) }\end{array}$ & p-value \\
\hline WBC, (G/L) & $5.3(5.1-6.0)$ & $6.5(5.0-7.6)$ & 0.142 \\
hsCRP, (mg/L) & $3.36(1.16-6.22)$ & $2.28(0.86-8.73)$ & 0.759 \\
se-ORM, (g/L) & $0.79(0.69-0.98)$ & $0.80(0.64-0.93)$ & 0.814 \\
u-TP, (mg/L) & $60(40-90)$ & $50(40-60)$ & 0.184 \\
u-CREAT, (mmol/L) & $8.55(5.83-12.41)$ & $9.54(7.70-13.95)$ & 0.654 \\
u-ORM, (mg/L) & $0.96(0.49-1.51)$ & $1.19(0.55-2.96)$ & 0.241 \\
u-ORM/u-CREAT, & $0.10(0.06-0.20)$ & $0.30(0.04-0.35)$ & 0.234 \\
(mg/mmol) & & & \\
\hline
\end{tabular}

Median (interquartiles) data are presented. WBC: white blood cell count; hsCRP: high sensitivity C-reactive protein; se-ORM: serum orosomucoid; u-TP: urinary total protein; u-CREAT: urinary creatinine; u-ORM: urinary orosomucoid

During subgroup-analyses, hsCRP and both serum and urinary ORM levels were found to be independent regard to age, gender and co-morbidities (obesity, diabetes, hypertension, COPD, etc.). Medications (angiotensin converting enzyme inhibitors, beta-receptor blockers, diuretics, antidiabetics, antiplatelet therapy, proton pump inhibitors, H2-receptor blockers) also showed no 
effects on u-ORM levels.

Significant positive correlation was found between $\mathrm{u}$-ORM/u-CREAT and hsCRP $(\mathrm{r}=0.26$, $\mathrm{p}=0.004)$; in addition between $\mathrm{u}-\mathrm{ORM} / \mathrm{u}-\mathrm{CREAT}$ and WBC ( $\mathrm{r}=0.189, \mathrm{p}=0.037)$ but none with se-ORM. Se-ORM correlated with hsCRP $(r=0.437 \mathrm{p}<0.001)$ and WBC $(\mathrm{r}=0.291, \mathrm{p}=0.003)$. Moreover hsCRP correlated with PASI $(r=0.245 . p=0.029)$.

\section{Discussion}

Psoriasis is a lifelong autoimmune disease affecting about $2-4 \%$ of the population in the Western world. It imposes high costs on the health-care system. It is also responsible for considerable psychosocial disability and negative effects on quality of life [1-3]. Monitoring of disease activity is essential for optimal treatment and for early recognition of complications, however controlling the skin symptoms is not sufficient since psoriasis is a systemic inflammatory condition. Laboratory tests, especially inflammatory markers may be helpful in clinical decision making.

The present study demonstrated that besides hsCRP, u-ORM could also be a useful, non- invasive marker in psoriasis.

To the best of our knowledge, this is the first study investigating u-ORM excretion in psoriasis. We found significantly higher u-ORM/u-CREAT levels in psoriatic patients compared to healthy individuals; moreover it might be an early and sensitive marker of psoriasis complications, since severe cases showed higher values, and u-ORM/u-CREAT was the only marker which was able to indicate joint inflammation; however this difference did not meet statistical significance.

To date several studies investigated the connection between psoriasis severity and CRP levels. According to the meta-analysis published by Dowlatshahi et al. CRP levels are significantly higher in patients suffering from psoriasis compared to healthy individuals [13]. However, most of the studies investigated patients with moderate or severe skin lesions (PASI $>12$ ) $[9,11,24,25]$. In our study, $64.4 \%$ of the patients had mild psoriasis (mean \pm SD PASI $6.52 \pm 7.11$ ); still, significantly higher hsCRP and $\mathrm{u}$-ORM/u-CREAT levels were found in psoriatic patients compared to healthy controls. This can be explained by the high sensitivity of these markers, while the less-sensitive se-ORM did not show any significant elevation in psoriasis. Nevertheless, Biljan et al. found significantly higher blood ORM levels in patients suffering from psoriasis when compared plasma ORM levels of 70 psoriatic patients to 40 healthy controls [16]. In the mentioned study, most of the involved patients had severe skin symptoms; extreme skin involvement was observed in $48.6 \%$ of the patients. In contrast, in our study the majority of the patients had mild psoriatic skin lesions se-ORM elevation. Seemingly, u-ORM is a more sensitive marker of inflammation than se-ORM, as it is capable of representing a low grade systemic inflammation in psoriatic patients.

The PASI is the most widely used tool for assessing psoriasis severity, however it describes only the skin symptoms of this systemic disease. Some laboratory tests are capable of indicating systemic inflammation thus supporting clinical decision making. In this study hsCRP correlated with PASI. Moreover significantly higher inflammatory parameters were found in severe cases (PASI>12), indicating the systemic manifestation of psoriasis.

Joint inflammation as severe systemic complication of psoriasis occurs about $30 \%$ of patients [26], however in our cohort only $8 \%$ of the patients developed psoriatic arthritis. As a sensitive marker, $\mathrm{u}-\mathrm{ORM} / \mathrm{u}$-CREAT levels were 3-fold higher in patients with psoriatic arthritis, but probably due to the low number of cases this result was not statistically significant.

Previous studies showed excessive u-ORM excretion in several chronic and acute inflammatory conditions [17-20]. Moderate elevation of u-ORM levels were found in diabetes mellitus and also in cardiovascular diseases, presumably associated with chronic low grade inflammation, endothelial dysfunction and oxidative stress which are also pathophysiologic factors in psoriasis [8, 22, 27-30].

It is well known that psoriatic patients have an increased risk of obesity, diabetes, cardiovascular diseases malignancies and other autoimmune diseases, these co-morbidities could influence u-ORM excretion. However, we did not find any differences regarding onset of diabetes, hypertension or other disorders and medications.

Furthermore, the pathomechanism of elevated $\mathrm{u}$-ORM excretion is not well clarified, although the correlations with inflammatory markers suggest that the systemic inflammation may be a crucial factor, as it has been supposed previously, too [17].

Limitations of the study are the low number of severe psoriatic cases. Moreover, relatively young individuals were selected as controls to reduce the risk of possible undiscovered diseases that could alter the concentrations of the investigated biomarkers.

\section{Conclusion}

As a highly sensitive, affordable and easily available non-invasive biomarker, urinary orosomucoid shows itself capable of becoming a 
novel inflammatory marker in psoriasis providing additional information on disease severity and progression. However, further investigations are needed to clarify its predictive value and exact role in the pathophysiology of psoriasis.

\section{Acknowledgments}

Péter Kustán and Balázs Németh were supported by the ÚNKP-17-3-III New National Excellence Program of the Ministry of Human Capacities, Hungary. The Authors would like to thank all the nurses of the Dermatology ward for their invaluable help. The present scientific contribution is dedicated to the 650th anniversary of the foundation of the University of Pécs, Hungary.

\section{Competing Interests}

The authors have declared that no competing interest exists.

\section{References}

1. Parisi R, Symmons DP, Griffiths CE, et al. Global epidemiology of psoriasis: a systematic review of incidence and prevalence. The Journal of investigative dermatology. 2013; 133: 377-85.

2. Griffiths CE, Barker JN. Pathogenesis and clinical features of psoriasis. Lancet. 2007; 370: 263-71.

3. Rapp SR, Feldman SR, Exum ML, Fleischer AB, Jr., Reboussin DM. Psoriasis causes as much disability as other major medical diseases. Journal of the American Academy of Dermatology. 1999; 41: 401-7.

4. Smith $\mathrm{CH}$, Barker JN. Psoriasis and its management. Bmj. 2006; 333: 380-4.

5. Shahwan KT, Kimball AB. Psoriasis and Cardiovascular Disease. The Medical clinics of North America. 2015; 99: 1227-42.

6. Huerta C, Rivero E, Rodriguez LA. Incidence and risk factors for psoriasis in the general population. Archives of dermatology. 2007; 143: 1559-65.

7. Nestle FO, Kaplan DH, Barker J. Psoriasis. The New England journal of medicine. 2009; 361: 496-509.

8. Peter I, Jagicza A, Ajtay Z, Kiss I, Nemeth B. [Psoriasis and oxidative stress]. Orvosi hetilap. 2016; 157: 1781-5.

9. Coimbra S, Oliveira H, Reis F, Belo L, Rocha S, Quintanilha A, et al. C-reactive protein and leucocyte activation in psoriasis vulgaris according to severity and therapy. Journal of the European Academy of Dermatology and Venereology: JEADV. 2010; 24: 789- 96

10. Isha, Jain VK, Lal H. C-reactive protein and uric Acid levels in patients with psoriasis. Indian journal of clinical biochemistry: IJCB. 2011; 26: 309-11.

11. Vadakayil AR, Dandekeri S, Kambil SM, Ali NM. Role of C-reactive protein as a marker of disease severity and cardiovascular risk in patients with psoriasis. Indian dermatology online journal. 2015; 6: 322-5.

12. Vanizor Kural B, Orem A, Cimsit G, Yandi YE, Calapoglu M. Evaluation of the atherogenic tendency of lipids and lipoprotein content and their relationships with oxidant-antioxidant system in patients with psoriasis. Clinica chimica acta; international journal of clinical chemistry. 2003; 328: 71-82.

13. Dowlatshahi EA, van der Voort EA, Arends LR, Nijsten T. Markers of systemic inflammation in psoriasis: a systematic review and meta-analysis. The British journal of dermatology. 2013; 169: 266-82.

14. Fournier T, Medjoubi NN, Porquet D. Alpha-1-acid glycoprotein. Biochimica et biophysica acta. 2000; 1482: 157-71.

15. Ceciliani F, Pocacqua V. The acute phase protein alpha1-acid glycoprotein: a model for altered glycosylation during diseases. Current protein \& peptide science. 2007; 8: 91- 108.

16. Biljan D, Situm M, Kostovic K, Batinac T, Matisic D. Acute phase proteins in psoriasis. Collegium antropologicum. 2009; 33: 83-6.

17. Kustan P, Szirmay B, Koszegi T, Ludany A, Kovacs GL, Miseta A, et al. Monitoring urinary orosomucoid in patients undergoing cardiac surgery: A promising novel inflammatory marker. Clinical biochemistry. 2017; 50: 1002-6.

18. Kustan P, Szirmay B, Horvath-Szalai Z, Ludany A, Lakatos A, Muhl D, et al Urinary orosomucoid: validation of an automated immune turbidimetric test and its possible clinical use. Biochemia medica. 2016; 26: 421-30.

19. Kustan P, Szirmay B, Horvath-Szalai Z, Ludany A, Kovacs GL, Miseta A, et al Urinary orosomucoid: a novel, early biomarker of sepsis with promising diagnostic performance. Clinical chemistry and laboratory medicine. 2017; 55: 299-307.
20. Park YJ, Yoo SA, Hwang D, Cho CS, Kim WU. Identification of novel urinary biomarkers for assessing disease activity and prognosis of rheumatoid arthritis. Experimental \& molecular medicine. 2016; 48: e211.

21. Svendstrup M, Christiansen MS, Magid E, Hommel E, Feldt-Rasmussen B. Increased orosomucoid in urine is an independent predictor of cardiovascular and all-cause mortality in patients with type 2 diabetes at 10 years of follow-up. Journal of diabetes and its complications. 2013; 27: 570-5.

22. Christiansen MS, Iversen K, Larsen CT, Goetze JP, Hommel E, Molvig J, et al. Increased urinary orosomucoid excretion: a proposed marker for inflammation and endothelial dysfunction in patients with type 2 diabetes. Scandinavian journal of clinical and laboratory investigation. 2009; 69: 272-81.

23. Schmitt J, Wozel G. The psoriasis area and severity index is the adequate criterion to define severity in chronic plaque-type psoriasis. Dermatology. 2005; 210: 194-9.

24. Chodorowska G, Wojnowska D, Juszkiewicz-Borowiec M. C-reactive protein and alpha2-macroglobulin plasma activity in medium-severe and severe psoriasis. Journal of the European Academy of Dermatology and Venereology: JEADV. 2004; 18: 180-3.

25. Serwin $A B$, Wasowicz W, Chodynicka B. Selenium supplementation, soluble tumor necrosis factor-alpha receptor type 1 , and C-reactive protein during psoriasis therapy with narrowband ultraviolet B. Nutrition. 2006; 22: 860-4.

26. Chandran V, Raychaudhuri SP. Geoepidemiology and environmental factors of psoriasis and psoriatic arthritis. Journal of autoimmunity. 2010; 34: J314-21.

27. El-Beblawy NM, Andrawes NG, Ismail EA, Enany BE, El-Seoud HS, Erfan MA. Serum and Urinary Orosomucoid in Young Patients With Type 1 Diabetes: A Link Between Inflammation, Microvascular Complications, and Subclinical Atherosclerosis. Clinical and applied thrombosis/hemostasis: official journal of the International Academy of Clinical and Applied Thrombosis/Hemostasis. 2016; 22: 718-26.

28. Hou LN, Li F, Zeng QC, Su L, Chen PA, Xu ZH, et al. Excretion of urinary orosomucoid 1 protein is elevated in patients with chronic heart failure. PloS one. 2014; 9: e107550.

29. Kadam DP, Suryakar AN, Ankush RD, Kadam CY, Deshpande KH. Role of oxidative stress in various stages of psoriasis. Indian journal of clinical biochemistry: IJCB. 2010; 25: 388-92.

30. Zhou Q, Mrowietz U, Rostami-Yazdi M. Oxidative stress in the pathogenesis of psoriasis. Free radical biology \& medicine. 2009; 47: 891-905. 